\title{
Excretory urography and renal scintigraphy for chronic obstructed kidney: does nonopacity mean nonsalvageability?
}

Alisa Klaipetch ${ }^{1}$, MD, Sirianong Namwongprom ${ }^{1}$, MD, Molrudee Ekmahachai ${ }^{1}$, MD, Bannakij Lojanapiwat ${ }^{2}$, MD

INTRODUCTION This study aimed to ascertain whether nonopacified kidney on excretory urography (also known as intravenous urography [IVU]) indicates nonsalvageability.

METHODS We retrospectively reviewed 45 adult patients with chronic unilateral urinary tract obstruction, in whom IVU revealed nonopacified kidney on one side but normal excretion on the contralateral side. Affected kidneys with split glomerular filtration rate (GFR) $<10 \mathrm{~mL} / \mathrm{min} / 1.73 \mathrm{~m}^{2}$ on ${ }^{99 \mathrm{mTC}} \mathrm{T}$-diethylenetriaminepentaacetic acid diuretic renal scintigraphy were considered nonsalvageable. Non-function was defined based on cutoff points $(<15 \%$ and $<20 \%$ ) to determine the sensitivity and specificity of differential renal function. Differences in IVU and renal scintigraphy findings, with respect to the duration of delayed filming on IVU, were analysed for significance.

RESULTS The results of IVU and renal scintigraphy findings for 34 (75.6\%) nonopacified kidneys matched, representing nonsalvageable kidneys. Sensitivity and specificity of differential renal function were $76 \%$ and $100 \%$, respectively, when the cutoff point for non-function was set at $<15 \%$. Sensitivity and specificity were $97 \%$ and $82 \%$, respectively, when the cutoff point was $<20 \%$. There was no significant difference between renal scintigraphy findings and IVU with 2 -hour and $>2$-hour delayed films $(p=0.96)$.

CONCLUSION Although most nonopacified kidneys on IVU were nonsalvageable, a quarter of them were found to be salvageable on renal scintigraphy. Besides split GFR, differential function at cutoff point $<15 \%$ could be used to determine non-function of a chronic obstructed kidney when the contralateral kidney is normal. Delayed filming beyond two hours appears unnecessary in ensuring non-excretion on IVU.

Keywords: chronic obstruction, differential renal function, excretory urography, nonsalvageable, renal scintigraphy

\section{INTRODUCTION}

Chronic unilateral urinary tract obstruction can lead to permanent loss of function of the affected kidney. Apart from the kidney being prone to traumatic rupture, the obstructed, non-functioning kidney may also act as a time bomb, where complications such as infection, stone formation, hypertension, intractable pain and bleeding are concerned. Nephrectomy or renal vascular embolisation may eventually be required to either resolve or prevent such complications. ${ }^{(1-4)}$ For this reason, evaluation of the residual function of an obstructed kidney is necessary in determining the proper management of such patients.

Among the many radiographic modalities currently available, simple excretory urography, also known as intravenous urography (IVU), remains the first-line investigation for both functional and structural evaluations of the kidney due to its availability and feasibility, as well as its properties that help in the evaluation of the anatomical and functional aspects of obstructed and unobstructed kidneys. ${ }^{(3)}$ However, IVU does not provide quantitative determination of renal function and is unable to obtain split renal function. Furthermore, some authors believe that IVU results are not straightforward and cannot be used to predict recoverability of the obstructed kidney. ${ }^{(5-7)}$ Therefore, renal scintigraphy using radionuclides continues to play an important role in solving this problem because of its unique properties, such as its ability to discriminate between mechanical obstruction and functional retention, and to provide qualitative and quantitative analyses of renal function. Total and split glomerular filtration rates (GFRs), in addition to the differential function of each kidney, can be obtained using renal scintigraphy. When IVU is indeterminate, using renal scintigraphy to assess decreased perfusion and function of the obstructed kidney is considered to be effective, reproducible and reliable. ${ }^{(8,9)}$ Chronic obstructed kidney with renographic GFR $<10 \mathrm{~mL} / \mathrm{min} / 1.73 \mathrm{~m}^{2}$ is widely accepted as nonsalvageable. ${ }^{(6,10)}$ Meanwhile, the threshold of differential function at which irreversible loss of renal function should be considered is usually accepted as $<15 \%-20 \%$. . $^{(3,11,12)}$

However, it is possible that clinicians may face situations where renal scintigraphy is unavailable (such as in small hospitals) and decisions on treatment depend on information gathered via IVU. Our study thus aimed to compare the accuracy of IVU against ${ }^{99 \mathrm{~m} T c-d i e t h y l e n e t r i a m i n e p e n t a a c e t i c}$ acid ( ${ }^{99 m}$ Tc-DTPA) diuretic renal scintigraphy in the determination of nonsalvageability of kidneys in patients with chronic obstruction.

\section{METHODS}

We retrospectively reviewed 45 adult patients diagnosed with chronic unilateral urinary tract obstruction due to stone retention

${ }^{1}$ Department of Radiology, Faculty of Medicine, ${ }^{2}$ Department of Surgery, Faculty of Medicine, Chiang Mai University, Chiang Mai, Thailand

Correspondence: Dr Alisa Klaipetch, Lecturer, Department of Radiology, Faculty of Medicine, Chiang Mai University, 110 Intavaroros Road, Amphur Muang, Chiang Mai 50200, Thailand. aklaipet@med.cmu.ac.th 
or ureteropelvic junction obstruction who underwent IVU and diuretic renal scintigraphy using ${ }^{99 \mathrm{~m} T c-D T P A}$ at the Chiang Mai University Hospital, Chiang Mai, Thailand, between January 2007 and September 2009. All patients had normal creatinine levels (range $0.6-1.4 \mathrm{mg} / \mathrm{dL}$ ). IVU findings studied over a period of at least two hours indicated no opacification of the affected kidney with no excretion of contrast medium, as well as normal excretory function of the contralateral kidney.

Standard IVU examinations were performed in the diagnostic radiology unit. All patients consumed at least two glasses of water $(500 \mathrm{~mL})$ and emptied the urinary bladder prior to the procedure. Intravenous contrast medium (Telebrix $350 \mathrm{mgl} / \mathrm{mL}$ or Omnipaque $300 \mathrm{mgl} / \mathrm{mL}$ ) at $1 \mathrm{~mL} / \mathrm{kg}$ of body weight was injected, and filming was performed 5 minutes, 15 minutes and approximately 30 minutes later. Delayed films were taken at least two hours following contrast administration for all patients with nonopacification of the affected kidney on routine imaging, with the maximum delay being four hours.

Diuretic renal scintigraphy was routinely performed using the same protocol of oral hydration $(500 \mathrm{~mL})$ within one month of IVU. An intravenous bolus injection of ${ }^{99 m}$ Tc-DTPA (dose $111 \mathrm{MBq}$ ) was given to each patient. After 15 minutes, $40 \mathrm{mg}$ of furosemide was administered intravenously. For image acquisition of a $128 \times 128$ frame, dynamic perfusion study was performed over 60 seconds at $1 \mathrm{~s} /$ frame, simultaneous to the radiopharmaceutical bolus injection. In the second phase of dynamic imaging, image was acquired at a rate of $10 \mathrm{~s} /$ frame for 30 minutes using the single-head, large field of view Phillips SKYLight gamma camera (Philips Medical Systems, Eindhoven, Netherlands) with a lowenergy, multipurpose collimator. The $20 \%$ energy window was centred at $140 \mathrm{keV}$.

The injected count was achieved by subtracting the count of the post-injection syringe from that of the pre-injection syringe, with decay correction. Regions of interest were manually drawn over each kidney, excluding the extrarenal pelvis. The image was processed, and the time-activity curve and quantitative data, including normalised GFR and split function of each kidney, were acquired using the JETStream ${ }^{\mathrm{TM}}$ acquisition system (Philips Medical Systems). Results were analysed and evaluated for renal perfusion, uptake, excretion, GFR and differential renal function of the affected kidney, as well as the contralateral side, via consensus review by two nuclear medicine physicians. Data on age, gender, side of affected kidney, body weight, serum creatinine level and the findings of diagnostic investigations (including IVU study time) were also collected. Results were abstracted from the patients' electronic medical records and the hospital database. A camera-based normalised split GFR value of $10 \mathrm{~mL} / \mathrm{min} / 1.73 \mathrm{~m}^{2}$ was used as the cutoff point to determine salvageability of the affected kidney. Nonopacified kidneys on IVU that had split GFR $<10 \mathrm{~mL} / \mathrm{min} / 1.73 \mathrm{~m}^{2}$ on renal scintigraphy were categorised as matched kidneys, while those on IVU that had split GFR $\geq 10 \mathrm{~mL} / \mathrm{min} / 1.73 \mathrm{~m}^{2}$ on renal scintigraphy were categorised as mismatched kidneys. IVU studies were
Table I. Patients' characteristics.

\begin{tabular}{lc}
\hline Characteristic & No. (\%) \\
\hline Age* $\left.^{*} \mathbf{y r s}\right)$ & $55.2 \pm 11.8(25-81)$ \\
Gender & $19(42)$ \\
$\quad$ Male & $26(58)$ \\
Female & $53.4 \pm 10.3(36-91)$ \\
Weight* (kg) & $15(33)$ \\
Affected kidney & $30(67)$ \\
Right & $1.1 \pm 0.2(0.6-1.4)$ \\
\hline Left & \\
Serum creatinine*
\end{tabular}

*Data is presented as mean \pm standard deviation (range).

Table II. Sensitivity and specificity of using two different cutoff points for differential function in ${ }^{99 m}$ Tc-DTPA diuretic scintigraphy in the determination of non-function.

\begin{tabular}{lcc}
\hline Cutoff point & Sensitivity (\%) & Specificity (\%) \\
\hline$<15 \%$ & 76 & 100 \\
$<20 \%$ & 97 & 82 \\
\hline
\end{tabular}

DTPA: diethylenetriaminepentaacetic acid

also categorised into two groups based on the duration of study needed to ensure that no contrast medium was excreted from the chronic obstructed kidney - those that required 2-hour or > 2-hour (range 2.5-4 hours) delayed filming before examination could be halted.

Continuous variables were described as means and standard deviation, whereas categorical variables were described in terms of percentages. Chi-square test with $95 \%$ confidence interval was used to determine statistical difference between the findings of delayed filming on IVU and those of renal scintigraphy.

\section{RESULTS}

The left kidney was affected in 30 of the 45 patients (67\%) with unilateral nonopacified kidney due to chronic obstruction. The patients' characteristics are presented in Table I. The sensitivities and specificities of the two different values of differential function used as cutoff points for non-function (using ${ }^{99 \mathrm{~m} T c-D T P A}$ diuretic renal scintigraphy) are shown in Table II. Nonopacified kidneys with differential function $<15 \%$ (with specificity of $100 \%$ ), which were found to consistently have split GFR $<10 \mathrm{~mL} / \mathrm{min} / 1.73 \mathrm{~m}^{2}$, were categorised as nonsalvageable kidneys.

IVU was performed with 2-hour delayed filming in 12 patients and > 2-hour delayed filming in 33 patients, before nonopacification was concluded. Table III shows the correlation between IVU and ${ }^{99 \mathrm{~m} T c-D T P A}$ renal scintigraphy findings in terms of matched or mismatched kidneys, based on the duration of delayed filming for IVU. Matched IVU and renal scintigraphy results were found in $34(75.6 \%)$ patients, while mismatched results were found in $11(24.4 \%)$ patients. No statistically significant difference was noted between renal scintigraphy findings and IVU studies with 2-hour and > 2-hour delayed films ( $p=0.96)$. 
Table III. Correlation between findings via excretory urography and renal scintigraphy.

\begin{tabular}{lrrr}
\hline Excretory urography & \multicolumn{2}{c}{ Renal scintigraphy* } & Total \\
\cline { 2 - 3 } & $\begin{array}{c}\text { Matched } \\
\text { results }\end{array}$ & $\begin{array}{c}\text { Mismatched } \\
\text { results }\end{array}$ & \\
\hline Duration of delayed filming & & & \\
2 hrs & $9(75.0)$ & $3(25.0)$ & 12 \\
$>2$ hrs & $25(75.7)$ & $8(24.3)$ & 33 \\
Total & $34(75.6)$ & $11(24.4)$ & 45 \\
\hline
\end{tabular}

\section{DISCUSSION}

IVU and renal scintigraphy are both widely accepted imaging tools for the determination of renal function in cases of chronic obstruction. Although renal scintigraphy is more advantageous than IVU in assessing quantitative function, its availability is limited, particularly in developing countries. IVU has come to the forefront because it is widely available, feasible and easy to perform, even in small rural hospitals that may lack nuclear medicine services. Although IVU is unable to provide quantitative information regarding the kidney's function, opacification and excretion seen on IVU can be considered signs of good function. When the kidney is not radiopaque and no excretion is observed on IVU, non-function is suspected and renal scintigraphy is usually needed to establish functionality. Several studies have reported on the role of radionuclide renal scintigraphy and its parameters in determining whether chronic obstructed kidney is recoverable. ${ }^{(9,11,12)}$ Split GFR $<10 \mathrm{~mL} / \mathrm{min} / 1.73 \mathrm{~m}^{2}$ on ${ }^{99 \mathrm{~m} T c-D T P A}$ renal scintigraphy has been widely accepted as an indicator of nonsalvageable kidney. ${ }^{(6,10)}$ In order to determine salvageability, the cutoff value for determining differential renal function has been accepted to be $<15 \%-20 \%$. $^{(3,11)}$ Our study also found excellent specificity when a differential function of $<15 \%$ was used as the cutoff point to detect non-function of the kidney. However, we found sensitivity to be higher when the cutoff point was set at a differential renal function of $20 \%$. Notwithstanding the above, the authors opine that using a differential function of $<15 \%$ as the cutoff point is more expedient, as it helps to avoid overestimation of non-function and overtreatment.

However, it is more appropriate and reliable to use split GFR than the percentage of differential renal function for determining split renal function, as the split GFR of one kidney is independent of its contralateral side. While split GFR represents the real function of the kidney of interest, regardless of the function of the contralateral kidney, the differential function of one kidney is dependent on the function of the contralateral kidney. Nevertheless, differential function would be reliable if the contralateral kidney is normal in terms of perfusion, uptake, excretory function, renogram curve and GFR. However, the differential function of the affected kidney would increase if the contralateral kidney has some degree of deteriorated function, leading to overestimation of the affected kidney's function. ${ }^{(13,14)}$ Thus, a chronic obstructed kidney with a differential function
$<15 \%$ can only be considered non-functional when the contralateral kidney is normal. Therefore, a combination of split GFR $<10 \mathrm{~mL} / \mathrm{min} / 1.73 \mathrm{~m}^{2}$ and differential renal function $<15 \%$ for chronic unilateral obstructed kidneys with normal contralateral sides can be confidently used as an indicator of non-function.

There was no definite conclusion with regard to the optimal duration of delayed filming on IVU for suspicion of very poor renal function. Earlier studies had recommended a waiting period of at least four hours for signs of excretion, in order to ensure that the affected kidney was no longer functional. Delayed filming as late as 24 hours after contrast injection has also been recommended. ${ }^{(5,15)}$ While many urologists and radiologists no longer adhere to such prolonged delayed filming, they believe that delayed filming for at least two hours should be carried out before examination can be halted. Many centres avoid waiting several hours for delayed films and instead, use ultrasonography to confirm hydronephrosis and thinning of the cortex of the nonopacified kidney. ${ }^{(16)}$ Alternatively, instead of emphasising the number of hours that delayed filming should be undertaken, focus should be placed on adequate hydration prior to the study. ${ }^{(17)} \mathrm{We}$, however, opine that the fewer the hours taken for IVU to accurately confirm non-excretion, the better. This reduced examination time is mainly beneficial to the patients. Relying on 2-hour delayed filming would reduce the duration of examination and personnel workload. We found that with adequate hydration, there was no significant difference between the results of 2-hour or $>$ 2-hour delayed filming. Our findings indicate that for IVU of chronic obstructed kidneys, the duration of study could be reduced to two hours, if adequate hydration is ensured.

Most nonopacified kidneys on IVU were nonsalvageable in our study, with IVU detecting $75.6 \%$ of nonfunctional kidneys. However, for a quarter of patients in whom the affected kidney was non-excreting on IVU (and would therefore be categorised as nonsalvageable), the nonopacified kidney on IVU was found to be salvageable on renal scintigraphy. Thus for these patients, diagnosis relying solely on IVU results might mean missed opportunities for proper management. More extensive studies with a larger number of patients are needed to confirm our findings.

In conclusion, our study found that in patients with unilateral chronic obstructed kidney, three-quarters of nonopacified kidneys on IVU were proven nonsalvageable on renal scintigraphy. However, renal scintigraphy findings also helped to prevent nearly a quarter of the kidneys from being incorrectly deemed as nonsalvageable based on IVU results alone. Besides split GFR $<10 \mathrm{~mL} / \mathrm{min} / 1.73 \mathrm{~m}^{2}$, setting the differential function at $<15 \%$ as a cutoff point for determining non-function of the affected kidney when the contralateral side is normal would also be useful. Among our cohort of patients with chronic unilateral urinary tract obstruction, there was no need for delayed filming beyond two hours on IVU in order to be certain that the affected kidney was not excreting. 


\section{REFERENCES}

1. Kinn AC. Ureteropelvic junction obstruction: long-term followup of adults with and without surgical treatment. J Urol 2000; 164:652-6.

2. Kubba AK, Hollins GW, Deane RF. Nephrectomy: changing indications, 1960-1990. Br J Urol 1994; 74:274-8.

3. Thomas HS. Hsu SS, Stephen YN. Management of Upper Urinary Tract Obstruction. In: Campbell MF, Walsh PC, Wein AJ, Kavoussi LR, eds. Campbell-Walsh Urology. 9th ed. Philadelphia: Saunders Elsevier, 2007: 1228-32.

4. Novick AC, Stream SB. Surgery of kidney. In: Campbell MF, Walsh PC, eds. Campbell's Urology. 6th ed. Philadelphia: Saunders, 1992: 2428-48.

5. Davidson AJ, Hartman DS, Choyke PL, et al. Obstructive uropathy. In: Davidson AJ, ed. Davidson's Radiology of the Kidney and Genitourinary Tract. 3rd ed. Philadelphia: Saunders, 1999: 201-2.

6. Shokeir AA, Provoost AP, Nijman RJ. Recoverability of renal function after relief of chronic partial upper urinary tract obstruction. BJU Int 1999; 83:11-7.

7. Bassiouny IE. Salvage pyeloplasty in nonvisualizing hydronephrotic kidney secondary to ureteropelvic junction obstruction. J Urol 1992; 148:685-7.

8. Carlsen O. The gamma camera as an absolute measurement device: determination of glomerular filtration rate in ${ }^{99 \mathrm{~m} T c-D T P A}$ renography using a dual head gamma camera. Nucl Med Commun 2004; 25:1021-9.

9. Fotopoulos A, Bokharhli]A, Tsiouris S, et al. [Comparison of six radionuclidic and non-radionuclidic methods for the assessment of glomerular filtration rate in patients with chronic renal failure.] Hell J Nucl Med 2006; 9:133-40. Greek.

10. Khalaf IM, Shokeir AA, El-Gyoushi FI, Amr HS, Amin MM. Recoverability of renal function after treatment of adult patients with unilateral obstructive uropathy and normal contralateral kidney: a prospective study. Urology 2004; 64:664-8.

11. Thomson A, Gough DC. The use of renal scintigraphy in assessing the potential for recovery in the obstructed renal tract in children. BJU Int 2001; 87:853-6.

12. Sreenevasan G. Bilateral renal calculi. Ann R Coll Surg Engl 1974; 55:3-12.

13. Maenhout A, Ham H, Ismaili K, et al . Supranormal renal function in the unilateral hydronephrosis: does it represent true hyperfunction? Pediatr Nephrol 2005; 20:1762-5.

14. Piepsz A, Ismaili K, Hall M, et al. How to interpret a deterioration of split function? Eur Urol 2005; 47:686-90

15. Tanagho EA, McAninch JW. Radiology of the urinary tract. In: Smith DR, Tanagho EA, McAninch JW, eds. Smith's General Urology. 16th ed. New York: McGraw-Hill, 2004: 65

16. Dunnick NR, Sandler CM, Newhouse JH, Amis ES. Textbook of Uroradiology. 4th ed. Philadelphia: Lippincott Williams \&Wilkins, 2007: 44

17. Kelalis PP, King LR, Belman AB. Clinical Pediatric Urology. 3rd ed. Philadelphia: Saunders, 1992: 701-3.

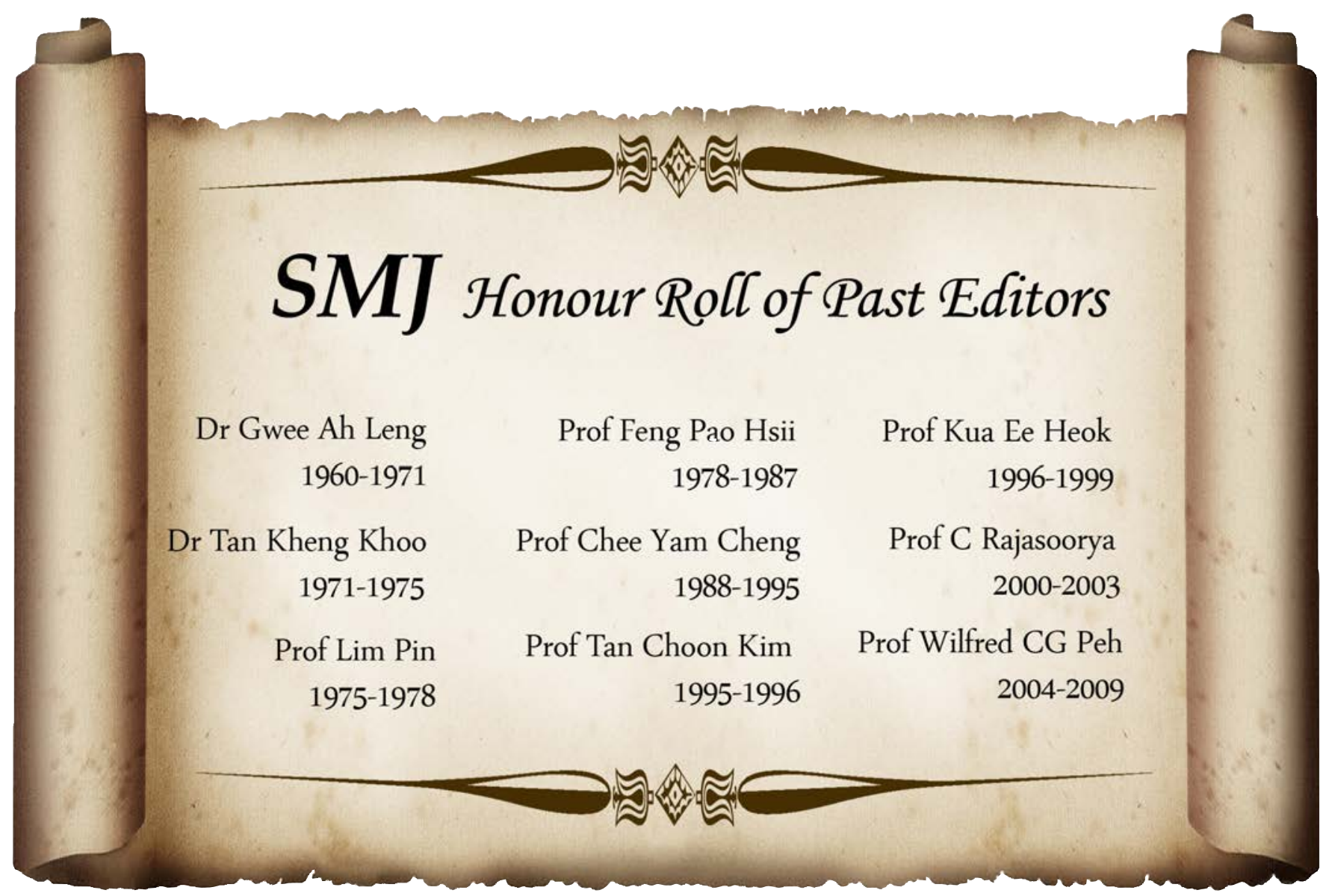

